\title{
Low-Voltage-Stress AC-Linked Charge Equalizing System for Series-Connected VRLA Battery Strings
}

\author{
Charnyut Karnjanapiboon $^{\dagger}$, Kamon Jirasereeamornkul ${ }^{*}$, and Veerapol Monyakul ${ }^{\text {** }}$ \\ $\dagger^{* *}$ Dept. of Electronic and Telecommunication Eng., King Mongkut’s University of Technology Thonburi (KMUTT), \\ Bangkok, Thailand \\ ${ }^{* *}$ Dept. of Pilot Plant Research and Development Unit (BEC), National Science and Technology Development \\ Agency (NSTDA), Pathumthani, Thailand
}

\begin{abstract}
This paper presents a low voltage-stress AC-linked charge equalizing system for balancing the energy in a serially connected, valve-regulated lead acid battery string using a modular converter that consists of multiple transformers coupled together. Each converter was coupled through an AC- linked bus to increase the overall energy transfer efficiency of the system and to eliminate the problem of the unbalanced charging of batteries. Previous solutions are based on centralized and modularized topologies. A centralized topology requires a redesign of the hardware and related components. It also faces a high voltage stress when the number of batteries is expanded. Modularized solutions use low-voltage-stress, double-stage, DC-linked topologies which leads to poor energy transfer efficiency. The proposed solution uses a low-voltage stress, AC-linked, modularized topology that makes adding more batteries easier. It also has a better energy transfer efficiency. To ensure that the charge equalization system operates smoothly and safely charges batteries, a small intelligent microcontroller was used in the control section. The efficiency of this charge equalization system is $85 \%$, which is $21 \%$ better than other low-voltage-stress DC-linked charging techniques. The validity of this approach was confirmed by experimental results.
\end{abstract}

Key words: Battery management system, Charge equalization system, Synchronous forward converter

\section{INTRODUCTION}

The demand for applications powered by valve-regulated lead acid (VRLA) batteries has increased rapidly [1], [2]. The advantage of VRLA batteries is the ability to distribute a high surge current to a load. However, a single VRLA battery unit is not enough to supply power to meet the demands of an overall system. Therefore, a serially connected battery string (SCBS) is required to support a huge surge in power for many applications. SCBSs are used in electric vehicles (EVs), Internet data centers (IDCs), telephone exchanges and uninterruptible power supplies (UPSs). Charging a serially connected battery string is simple because a single power source is required to connect at the bottom-end and the top-end electrical terminals of the battery stack. Unfortunately, the

Manuscript received Sep. 30, 2012; revised Dec. 15, 2012

Recommended for publication by Associate Editor Woo-Jin Choi.

${ }^{\dagger}$ Corresponding Author: chanyut@rmutl.ac.th

Tel: +662-470-9070, Fax: +6654-711-601, KMUTT

*Dept. of Electronic and Telecommunication Eng., King Mongkut's University of Technology Thonburi (KMUTT), Thailand

** Dept. of Pilot Plant Research and Development Unit (BEC), National Science and Technology Development Agency (NSTDA), Thailand
VRLA batteries in a string are not completely uniform, even when they were produced on the same production line. Small differences in the chemical energy stage of the batteries may cause an unequal charge in any one battery in a stack when charging and discharging, creating an unbalanced effect. This in turn results in either overcharging or undercharging and reduces the system's reliability. The unbalance causes the stack to age prematurely and shortens its service life. Of greater concern is the safety of the system. An explosion can occur due to rapid hydrogen gas production during overcharging. To manage this imbalance effect, a cell charge equalizing system (CES) is used. A CES is an important part of a battery management system (BMS). A CES is composed of many charge equalization circuits (CECs). A CEC is responsible for moving the excess energy from a source battery to a target battery.

From previous research on CESs, there are several equalizing techniques available, and these are shown in Fig. 1. To control the excess energy of a fully charged battery, Refs. [3]-[6] proposed dissipative techniques that use an individual cell equalizer (ICE) consisting of one resistor and one switch 


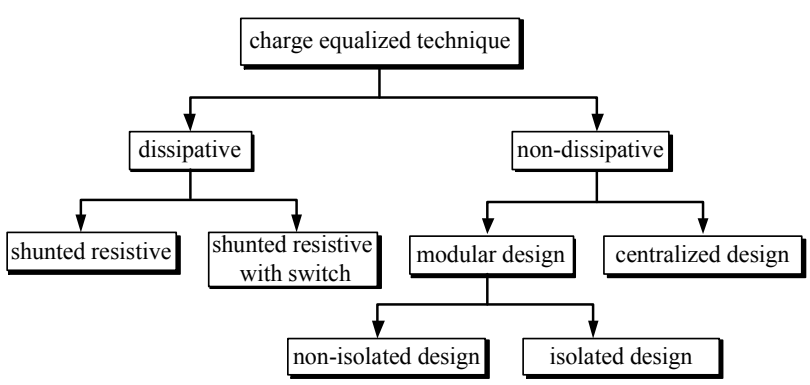

Fig. 1. Diagram of the charge equalization technique used in CESs.

connected in parallel to each battery in a string.

The efficiency of this technique is extremely low because all of the excess energy is changed into heat through the resistor. To improve the system's efficiency, non dissipative techniques have been developed [7]-[36]. These techniques takes advantage of a power electronic converter to transport excess energy from a source battery to a target battery. The operation of a non dissipative technique can be categorized into two main topologies: centralized design and modular design. In the centralized designs [7]-[9] a single-direction, multi-winding technique was introduced. An additional power source was required to supply more charging current to the SCBS. It also flows synchronously into the battery. However, if a battery has been fully charged, this topology can not handle an overcharge of that battery.

Ref. [10] proposed an additional switch for the control of the additional charging current. This switch was placed on a conductor connecting a multi wound transformer and a battery in an SCBS. Ref. [11] introduced a centralized bidirectional charge equalization circuit to supply an additional charging current to a hungry battery and to drain the excess energy from a fully charged battery.

Refs. [12]-[16] proposed a switch capacitor system to equalize the energy of the batteries that are closest together. This technique led to the design of a complicated switch driver circuit. If the voltages of the batteries were significantly different, the capacitor current would have a large value and would cause damage to the switch.

Ref. [17] introduced a non-dissipative balanced charging using a Ćuk topology. This technique involves passing excess energy in two directions among the nearest-neighbor batteries.

Ref. [18] introduced a non-dissipative balanced charging using a buck-boost topology. This technique involves passing excess energy in the top-down direction. In the last battery unit at the bottom, the additional buck-boost converter transferred the energy to a common DC charging bus at a very high voltage. As a result, the switch faced a high stress voltage. Therefore, the system reliability decreased.

Ref. [19] introduced a low-stress, non-dissipative balanced charging using a buck-boost topology to correct the problem noted in the previous study. As in the previous technique, the equalization was in the top-down direction. The balancing period could take several charging periods. A bidirectional converter was proposed by Refs. [17] and [20], [21] using CECs in both directions: top-down and bottom-up. The aim of this topology was to manage the excess energy of two batteries placed closely together.

A modularized charge equalization converter was presented with the CES designed as a modular package, [9] and [22]-[30]. Refs. [31]-[33] introduced a DC-linked technique to achieve an improvement in the efficiency of the transfer of energy between battery modules in an SCBS with a modular design.

Even though these techniques were able to increase the total transfer efficiency of the CES, they operated in a double-stage manner. The first stage was used to carry the excess energy from the fully charged battery to the DC-linked bus. The second stage was used to carry the DC-linked energy to another battery in the string. The efficiency was moderate because the total transfer energy was a result of multiplying the two converter efficiencies. To better understand these traditional balancing processes, a three-dimensional efficiency transfer graph was analyzed [31].

Ref. [34] proposed a technique for balancing energy by using a flyback converter with multiple-wound transformers. This technique was centralized and not modular. Ref. [35] proposed a multi-flyback transformer with an inter-module equalizer. Ref. [36] proposed a multi-flyback transformer without an inter-module equalizer. Some of the power switching devices in these designs [34]-[36] experience a high-voltage stress on the top battery terminal (more batteries equal more voltage stress).

Both topology types (centralized and modularized) take the same amount of energy, but when the size of the system needs to be extended to accommodate additional units, the centralized topology makes it difficult to insert and remove converter modules from the CES. In changing the number of batteries in the SCBS, the centralized topology also requires a redesign of the hardware and related components. Meanwhile, with the modularized topology, only a small change in the microcontroller software is required. In the centralized topology, the voltage stress of the switch depends on the number of batteries in the SCBS unit. When the size of the SCBS system increased, additional battery units are used. This leads to a higher voltage stress developing across the active switches. This is due to the fact that the centralized topology requires electrical power from the high voltage present at the main charging terminals.

The presented work describes a modular single-stage low-voltage-stress $\mathrm{CEC}$ with an AC-linked bus technique to increase the overall transfer efficiency among the batteries in a SCBS. The modular design of the proposed scheme makes it possible for an engineer to easily fix circuit problems during maintenance. 


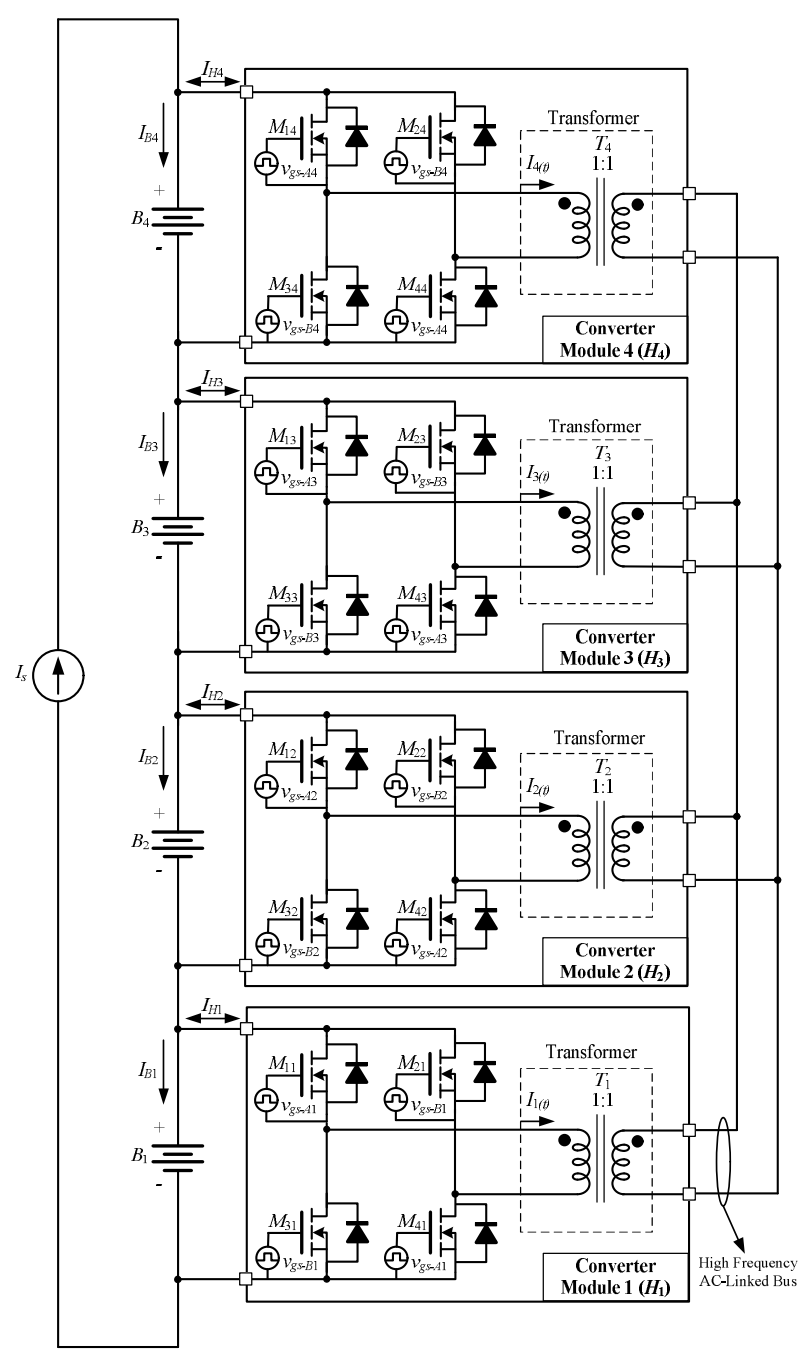

Fig. 2. Diagram of the proposed circuit.

\section{The Proposed CHARgE EQUALIZATION TECHNIQUE}

A block diagram of the proposed system is shown in Fig. 2. The key concept is how to transfer excess energy to a target battery as quickly as possible by using the property of electromagnetic coupling. Unlike the previous CEC technique, the proposed CEC consisted of modular bidirectional DC-DC converters connected through a high-frequency AC-linked bus. A current source $\left(I_{s}\right)$ supplied a constant current for charging the batteries of the SCBS. Each converter module is represented by $H_{n}$. The bidirectional current that flowed between the batteries and the converters is called $I_{H n}$.

The number of CEC modules was equal to the number of batteries in the SCBS. Each of the converter modules was connected to the associated battery units. The details of the single-stage forward converter of the CES module are shown in Fig. 3. It consisted of four switches and one transformer. The CES module had a low component count, and it was operated at low-voltage-stress switching to give the highest operational

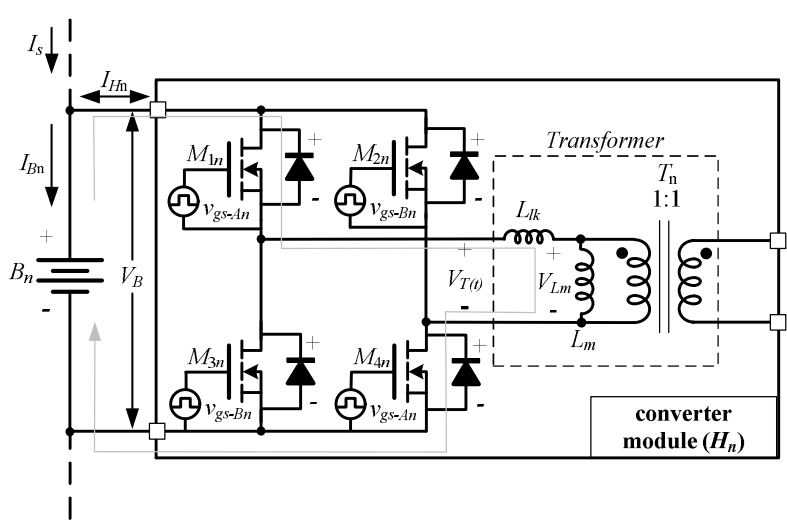

Fig. 3. Detailed diagram of the proposed charge equalization modules.

reliability. The transformer core was assembled with a $0.1 \mathrm{~mm}$ air gap wiring. The leakage inductance of the transformer was $1.5 \mu \mathrm{H}$. The total energy from the primary side was allowed to transfer to the secondary side through the transformer. The turn ratio of each transformer was 1:1. The MOSFETs $M_{1 n}$ with $M_{4 n}$ and $M_{2 n}$ with $M_{3 n}$ were alternately turned on and off.

\section{OPERATION OF THE SINGLE-STAGE SYNCHRONOUS FORWARD CES CONVERTER}

From a diagram of the CES (Fig. 2) and the details for each converter module $\left(H_{n}\right)$ (Fig. 3), the essential operating modes are presented in Fig. 4 and Fig. 5, respectively. An AC-linked bus operates as a shortcut path for the excess energy of a fully charged battery cell. In the charging process of a serially connected battery, the stage of charge (SOC) is proportional to the battery voltage, so the responsibility of the CES is to ensure that the voltages of each battery are equal. This responsibility was accomplished by equalizing the excess energy of one battery to make it equal to another battery. To do this, it is assumed that $V_{B 1}, V_{B 2}, V_{B 3}$ and $V_{B 4}$ are $7 \mathrm{~V}, 6.5 \mathrm{~V}, 6 \mathrm{~V}$ and 7.5 $\mathrm{V}$, respectively. The operation of the proposed technique can be described in five stages. The details are as follows.

First stage $\left(t_{0}-t_{1}\right)$ : switches $M_{1 n}$ and $M_{4 n}$ were turned on synchronously. $M_{2 n}$ and $M_{3 n}$ were turned off. Some of the energies from batteries $B_{1}, B_{2}, B_{3}$ and $B_{4}$ are interchanged and some of the energies are stored in each transformer at a magnetizing inductance $\left(L_{m}\right)$ and leakage inductance $\left(L_{l k}\right)$. In this stage, the voltage drop across the switches $M_{1 n}$ and $M_{4 n}$ equaled the product of the switches' turn-on resistance $R_{D S_{-} \text {on }}$ and transformer currents $I_{n(t)}$. The currents from batteries $B_{4}$ and $B_{1}$ negatively increased, but the currents from batteries $B_{3}$ and $B_{2}$ positively increased. The negative current flow waveform meant that the excess energy flowed from the battery into the converter. The positive current flow waveform meant that additional current was supplied to each battery. In this region, the current from battery $B_{3}$ was greater than that from the other batteries because battery $B_{3}(6 \mathrm{~V})$ had the lowest 
voltage. The excess energy from batteries $B_{4}(7.5 \mathrm{~V})$ and $B_{1}(7$ V) was transferred to battery $B_{3}$. Battery $B_{2}$ took less current than battery $B_{3}$ because the voltage of battery $B_{2}$ was higher than that of battery $B_{3}$.

Second stage $\left(t_{1}-t_{2}\right)$ : switches $M_{1 n}$ and $M_{4 n}$ were turned off. $M_{2 n}$ and $M_{3 n}$ were synchronously turned on. The voltage drop across the switches were equal to the battery voltage in the clamping mode, which maintained a low-voltage stress for the proposed CEC. The energy from $L_{m}$ and $L_{l k}$ of each transformer decreased, being transferred back to the batteries. The current amplitude of $B_{4}, B_{3}, B_{2}$ and $B_{1}$ decreased. The amplitude of this current depended on the battery voltage. A lower voltage would produce a high current amplitude.

Third stage $\left(t_{2}-t_{3}\right)$ : switches $M_{1 n}$ and $M_{4 n}$ were kept turned off. $M_{2 n}$ and $M_{3 n}$ were still turned on, except for $M_{24}$ and $M_{34}$, which were turned off. After the energy from $L_{m}$ and $L_{l k}$ of the transformer dropped to zero, the voltage drop across the switches $M_{1 n}$ and $M_{4 n}$ was again equal to that of the second stage. The current of battery $B_{4}$ was zero. The currents of batteries $B_{3}$ and $B_{2}$ decreased. The current of battery $B_{1}$ decreased to zero.

Fourth stage $\left(t_{3}-t_{4}\right)$ : switches $M_{1 n}$ and $M_{4 n}$ were turned off. $M_{2 n}$ and $M_{3 n}$ were still turned on, except for $M_{22}, M_{32}, M_{23}$ and $M_{33}$, which were turned off. The currents of batteries $B_{4}$ and $B_{1}$ were zero. The current of battery $B_{3}$ decreased. The current of battery $B_{2}$ became zero.

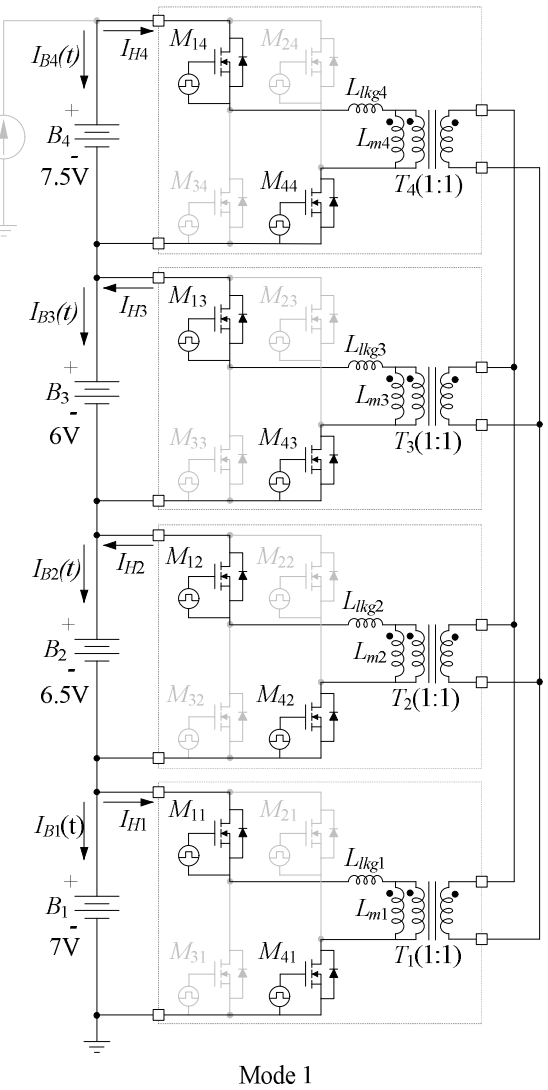

Fig. 4. Operation modes of the proposed technique.
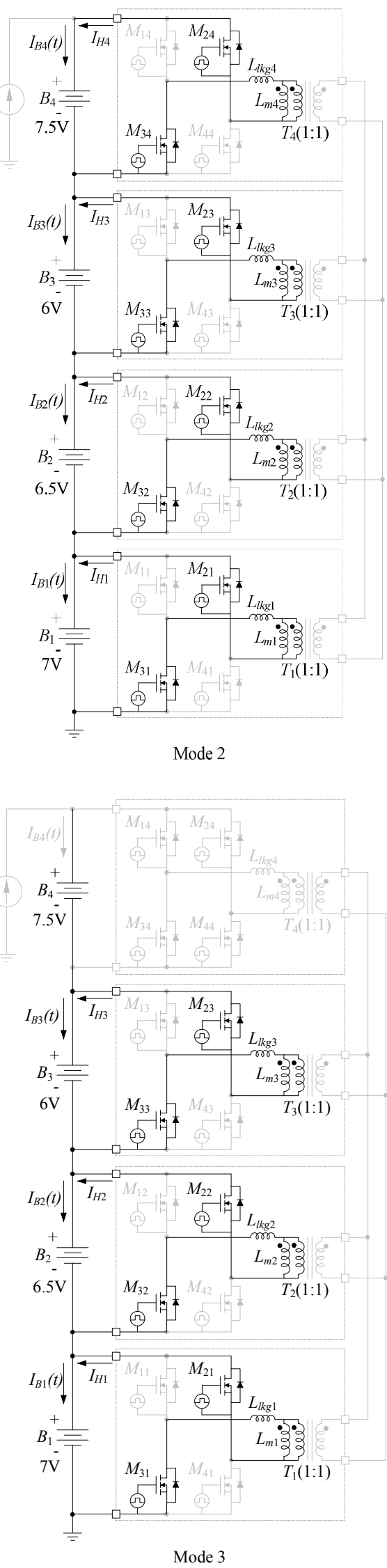

Fig. 4. Operation modes of the proposed technique (cont'd). 

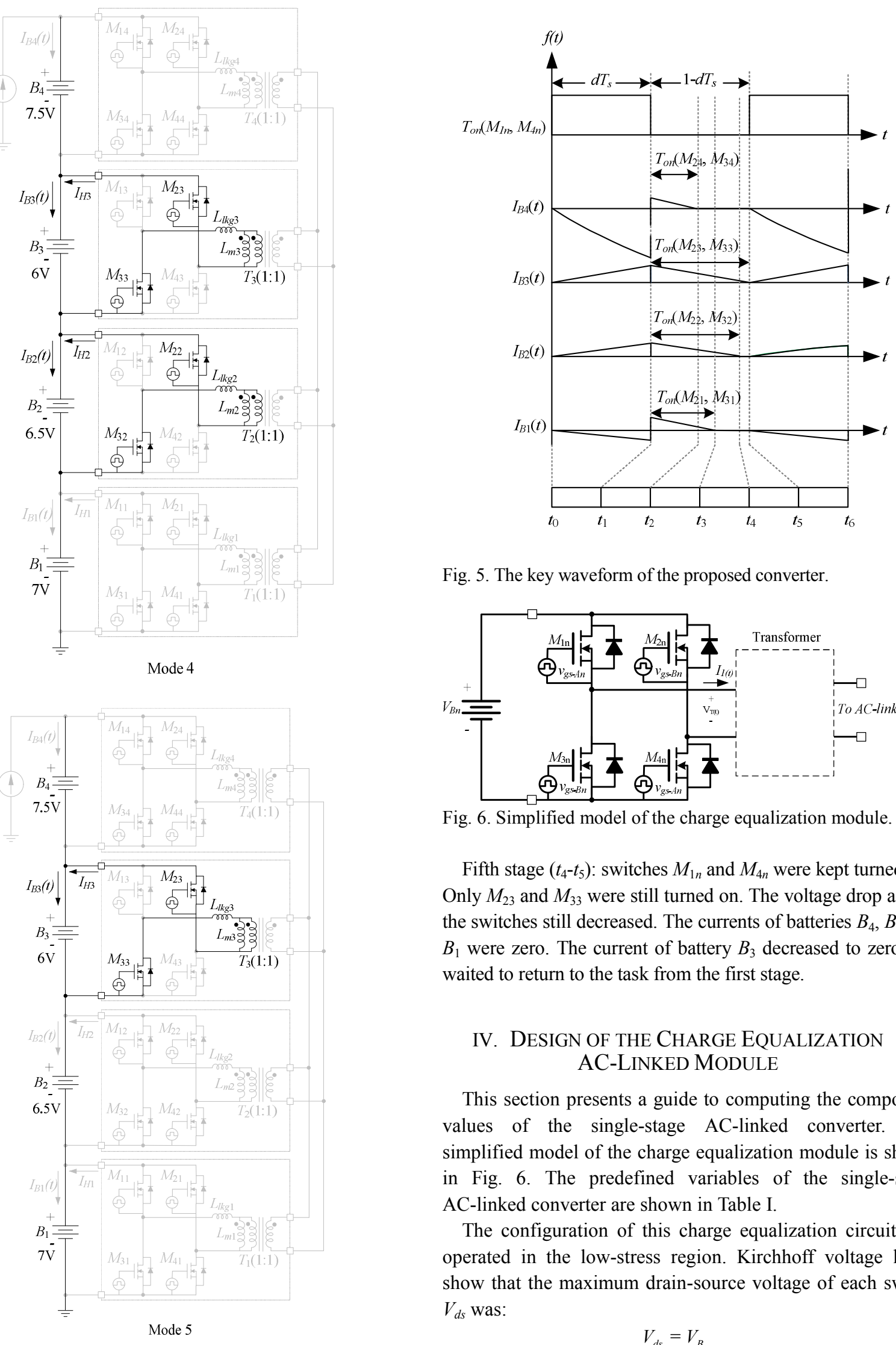

Fig. 5. The key waveform of the proposed converter.

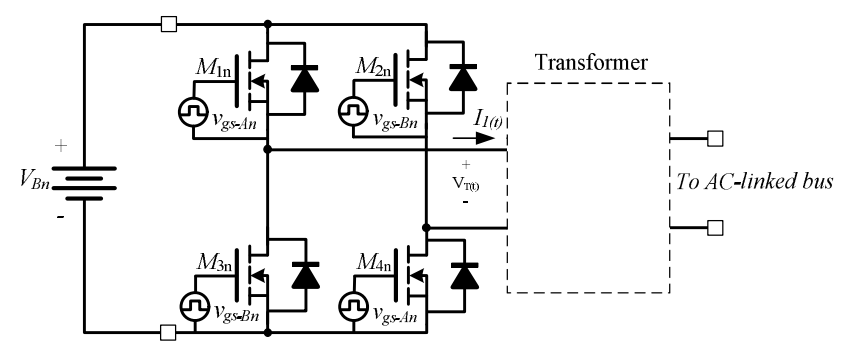

Fig. 6. Simplified model of the charge equalization module.

Fifth stage $\left(t_{4}-t_{5}\right)$ : switches $M_{1 n}$ and $M_{4 n}$ were kept turned off. Only $M_{23}$ and $M_{33}$ were still turned on. The voltage drop across the switches still decreased. The currents of batteries $B_{4}, B_{3}$ and $B_{1}$ were zero. The current of battery $B_{3}$ decreased to zero and waited to return to the task from the first stage.

\section{DESIGN OF THE CHARGE EQUALIZATION AC-LINKED MODULE}

This section presents a guide to computing the component values of the single-stage AC-linked converter. The simplified model of the charge equalization module is shown in Fig. 6. The predefined variables of the single-stage AC-linked converter are shown in Table I.

The configuration of this charge equalization circuit was operated in the low-stress region. Kirchhoff voltage loops show that the maximum drain-source voltage of each switch $V_{d s}$ was:

$$
V_{d s}=V_{B}
$$

Fig. 4. Operation modes of the proposed technique (cont'd). 
TABLE I

Predefined Variables of the Single-Stage AC-LINKED CONVERTER

\begin{tabular}{l|l}
\hline Input variable & Value \\
\hline Number of VRLAs in the SCBS & 4 Units \\
\hline Maximum input voltage $\left(V_{\text {in_max }}\right)$ & $7.5 \mathrm{~V}$ \\
\hline Minimum input voltage $\left(V_{\text {in_min }}\right)$ & $6.0 \mathrm{~V}$ \\
\hline Nominal input voltage $\left(V_{\text {in_norm }}\right)$ & $7.0 \mathrm{~V}$ \\
\hline Power output $\left(P_{o}\right)$ & $6.0 \mathrm{~W}$ \\
\hline Charging current $\left(I_{s}\right)$ & $0.8 \mathrm{~A}$ \\
\hline Switching frequency $\left(f_{s w}\right)$ & $20 \mathrm{kHz}$ \\
\hline Transformer coefficient $(\eta T r)$ & 0.99 \\
\hline MOSFET turn-on resistance $\left(R_{d s \_o n}\right)$ & $0.028 \Omega$ \\
\hline
\end{tabular}

For a maximum battery voltage of $7.5 \mathrm{~V}$, the maximum $V_{d}$ is equal to $7.5 \mathrm{~V}$.

Therefore, it was possible to use a low-voltage switch in the charge equalization module. Under low-voltage operation of the main power switch, the system reliability was high. While the switch was operated, the maximum drain source voltage was calculated by:

$$
\begin{aligned}
& V_{d s_{-} o n}=\frac{P_{o}}{\eta T r \times V_{\text {in } \min }} \times R_{d s_{-} o n} \\
& V_{d s_{-} o n}=\frac{6.0}{0.99 \times 6.0} \times 0.028 \simeq 0.028 \mathrm{~V}
\end{aligned}
$$

The transformer functioned as a magnetic coupling, implying that the transformer turn ratio $\left(N_{P S}\right)$ could be equal to one. The primary magnetizing inductance voltage was computed.

$$
\begin{aligned}
& V_{L m}=N_{P S} \times\left(V_{B_{-} \max }+\left(2 \times V_{d s_{-} \text {on }}\right)\right) \\
& V_{L m}=1 \times(7.5+(2 \times 0.028))=7.556 \mathrm{~V}
\end{aligned}
$$

The maximum turn-on time was calculated.

$$
\begin{aligned}
& T_{\text {on_ } \max }=\frac{V_{L m} \times T}{\left(V_{\text {in_ } \left.\min -\left(2 \times V_{d s_{-} \text {on }}\right)\right)+V_{L m}}\right.} \\
& T_{\text {on } \max _{-}}=\frac{7.556 \times 50 \times 10^{-6}}{(6-(2 \times 0.028))+7.556}=27.98 \mu \mathrm{s}
\end{aligned}
$$

The minimum turn-on time was calculated.

$$
\begin{aligned}
& T_{o n_{-} \min }=\frac{V_{L m} \times T}{\left(V_{\text {in_ } \max }-\left(2 \times V_{d s_{-} o n}\right)\right)+V_{L m}} \\
& T_{\text {on }_{-} \min }=\frac{7.556 \times 50 \times 10^{-6}}{(7.5-(2 \times 0.028))+7.556}=25.19 \mu \mathrm{s}
\end{aligned}
$$

The maximum duty cycle was calculated.
TABLE II

THE COMPONENT VARIABLES OF THE CONVERTER

\begin{tabular}{l|l}
\hline Component Name & Value \\
\hline MOSFET $\left(M_{1 n}, M_{2 n}\right)$ & IRFZ44N \\
\hline Synchronous switch $\left(D_{1 n}, D_{2 n}\right)$ & IRFZ44N \\
\hline Transformer inductance $\left(L_{p}\right)$ & $180 \mu \mathrm{H}$ \\
\hline Transformer turn ratio & $1: 1$ \\
\hline
\end{tabular}

$$
D_{\max }=\frac{T_{\text {on_max }_{-}}}{T}=\frac{27.98 \times 10^{-6}}{50 \times 10^{-6}}=0.559
$$

The minimum duty cycle was calculated.

$$
D_{\min }=\frac{T_{\text {on_min }}}{T}=\frac{25.19 \times 10^{-6}}{50 \times 10^{-6}}=0.503
$$

The primary current ramp amplitude was calculated

$$
\begin{aligned}
& \Delta I_{p a}=\frac{2 \times P_{o}}{\left(V_{\text {in_min }}-\left(2 \times V_{d s_{\_} o n}\right)\right) \times \eta \times D_{\max }} \\
& \Delta I_{p a}=\frac{2 \times 6}{(6-(2 \times 0.028)) \times 0.99 \times 0.559}=3.648 \mathrm{~A} \\
& L_{p}=\frac{\left(V_{\text {in_min }}-\left(2 \times V_{d s_{-} o n}\right)\right)}{\Delta I_{p a}} \times T_{o n_{-} \max } \\
& L_{p}=\frac{(6-(2 \times 0.028))}{3.648} \times 27.98 \times 10^{-6}=45.59 \mu \mathrm{H}
\end{aligned}
$$

Each magnetizing inductance $\left(L_{m}\right)$ of the CEC's output transformers was linked in a parallel connection. Thus the inductance of each transformer in the CEC modules for four-unit VRLA batteries could be calculated.

$$
L_{m}=n_{H} \times L_{p}=4 \times 45.59 \mu \mathrm{H}=182.36 \mu \mathrm{H}
$$

Where $n_{H}$ is the number of active CES modules.

From Eq. (1)-(10), the component variables of the charge equalization module were calculated, and their values are given in Table II.

\section{CONTROLling PRINCIPLE}

A control diagram of the proposed system is shown in Fig. 7. In this prototype, there were four battery units connected in a serial pattern. Thus the number of CEC modules should be equal to the number of battery units, yielding four CEC modules. The four CEC modules were $H_{1}, H_{2}, H_{3}$ and $H_{4}$.

The main control system used a 16-bit microcontroller unit (MCU) with a computation speed of 30 mega-instructions per second (MIPS). The voltage of each battery was sent to the MCU individually via an isolated signal-conditioning circuit. 


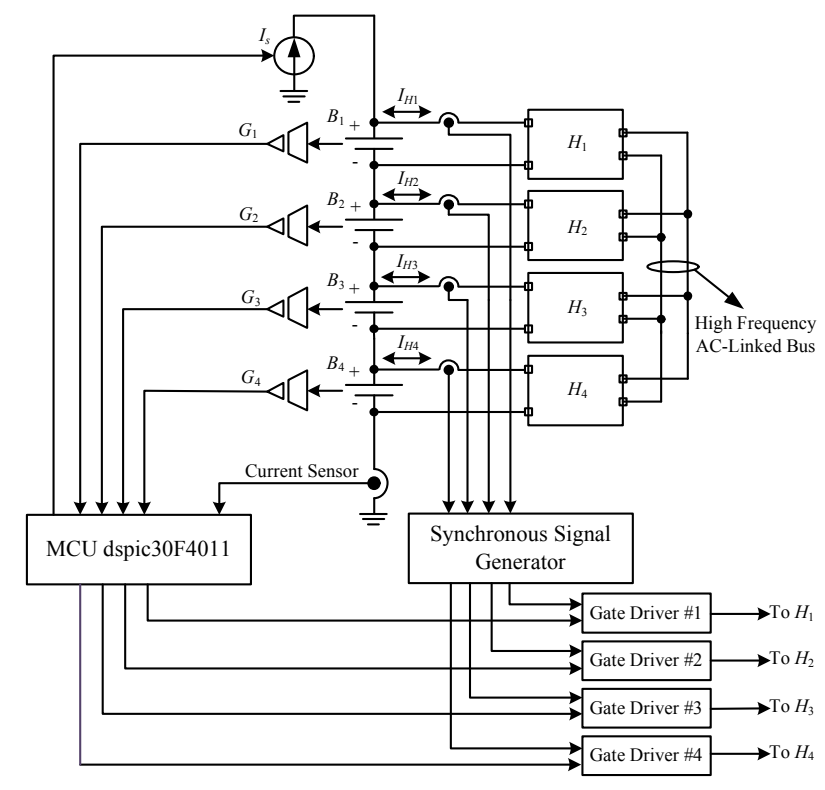

Fig. 7. Diagram of the proposed CEC technique.

The battery output signals were $G_{1}, G_{2}, G_{3}$ and $G_{4}$. These signals were sent to the MCU for computing and generating gate-driving signals for the CEC modules. After the MCU internally completed and generated the gate-driving signal in a pulse width modulation (PWM) style, the MCU gave a voltage signal to the gate driver circuit. This isolated gate driver circuit generated the proper voltage for controlling the main active switch in the CEC module. The synchronous signal generator was a system that measures the transformer current of a converter module and then generates a synchronous-command signal to switches $D_{2 n}$ and $D_{3 n}$ to increase the total energy transfer efficiency. The current sensor supplied the charging current information to the MCU. When it is finished charging a battery, the MCU sends an inhibition signal to disable the charging current source $\left(I_{s}\right)$.

To scale up the control scheme for a larger number of units in the SCBS, the main centralized control MCU and related components were changed to a localized control MCU. The localized control MCU was constructed by embedding a control MCUs in each module of the charge equalized converters, linked together via a data communication channel. After the MCU completed communicating the data, it computed an appropriate compensation command and then sent this command to each charge equalized converter to balance the energy of the VRLA batteries.

\section{EXPERIMENTAL RESULTS}

Six volt VRLA batteries were selected because the voltage of commercially available VRLA batteries is at the medium range of $2 \mathrm{~V}$ to $12 \mathrm{~V}$. The aim of this experiment was to verify the low-voltage-stress charge equalizing system with medium voltage VRLA batteries. However, the proposed technique can also be applied to $48 \mathrm{~V}$ telecommunication

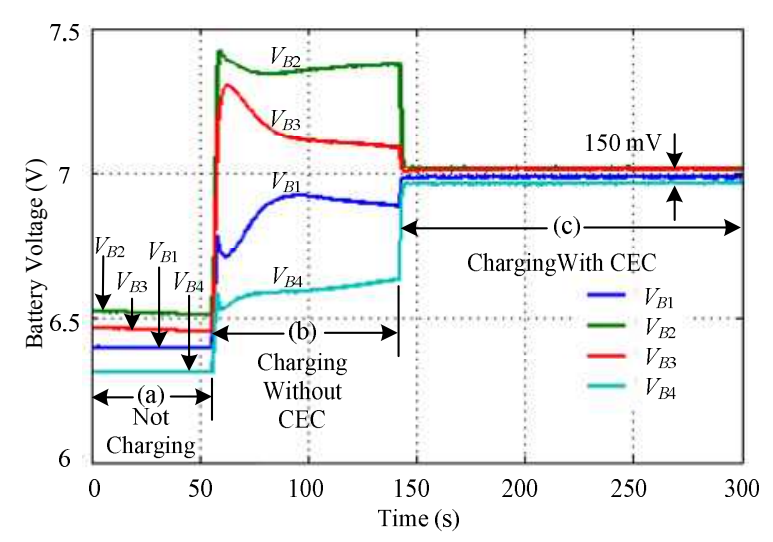

Fig. 8. The relationship of battery voltage and charging time.

systems and $125 \mathrm{~V}$ substation systems.

To verify the validity of proposed technique, four battery units with four different stages of charge were used. Each battery was connected together in a serial configuration. A uniform power source fed the same charging current to each of these batteries. Using a CEC assured a controlled and uniform voltage in the charging of each of the batteries. A 22-bit resolution PC recorded the battery voltage data. A laboratory power supply (Model GPS-4303, GWINSTEK) provided the charge to the SCBS; an oscilloscope (DS1022C, RIGOL) measured the converter's electrical signal waveforms; a true RMS digital multimeter (FLUKE179, FLUKE) monitored the battery voltages; a digital multimeter (MODEL 1009, KYORITSU) measured the efficiency of the proposed system; and a SL6-5 battery (SPA ${ }^{\circledR}$ VRLA battery 6 $\mathrm{V} / 5 \mathrm{Ah}$ ) was the test subject.

The operating frequency of the CEC system was $20 \mathrm{kHz}$. The initial conditions of the four batteries at different stages of charge (SOC) were $B_{1}=6.37 \mathrm{~V}, B_{2}=6.52 \mathrm{~V}, B_{3}=5.47 \mathrm{~V}$ and $B_{4}=6.31 \mathrm{~V}$.

To manage the unbalanced individual energies in the battery string, CESs were employed. Fig. 8 shows the battery voltages while charging with the CESs.

The initial stage is depicted in the beginning region (a), and it shows that each battery started at a different voltage. The voltages of the batteries are arranged from highest to lowest: $V_{B 2}, V_{B 3}, V_{B 1}$, and $V_{B 4}$. The stage of charging without the CES is shown in region (b). Each battery voltage is increasing uniformly. In region (c), the proposed CES handles the equalization process to equalize the battery voltage. The voltages of the batteries are conditioned together with a voltage range of $150 \mathrm{mV}$. At the end of the charging process, each battery has approximately the same voltage $(7 \mathrm{~V})$.

The oscillograms of the proposed CEC are shown in Fig. 9. The initial conditions of the four batteries were $B_{1}=5.94 \mathrm{~V}$, $B_{2}=6 \mathrm{~V}, B_{3}=5.61 \mathrm{~V}$ and $B_{4}=6.23 \mathrm{~V}$.

The current of the highest-voltage battery, $B_{4}$, is shown in Fig. 9(a). The current of the battery with the lowest voltage, 


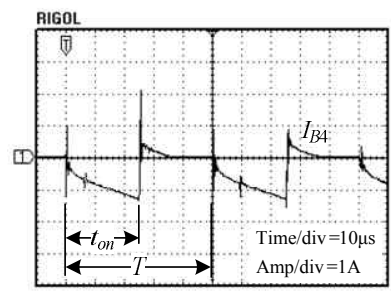

(a) The current of the highest voltage battery, $B_{4}$

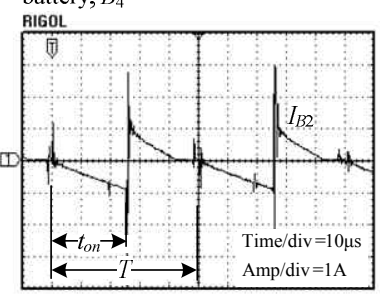

(c) The current of the batteries with moderate voltage, $B_{2}$

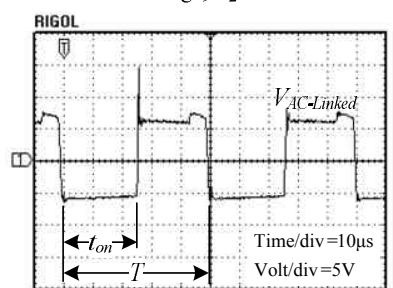

(e) The AC-linked voltage at the interconnection bus

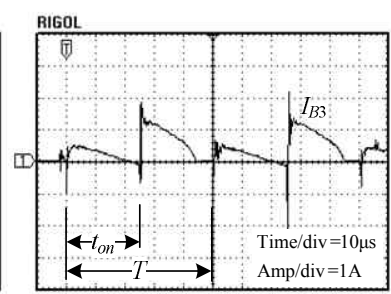

(b) The current of the battery with the lowest voltage, $B_{3}$

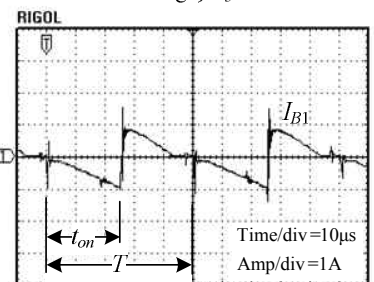

(d) The current of the batteries with moderate voltage, $B_{1}$

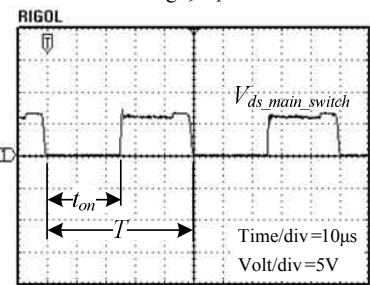

(f) The voltage stress drop across the main active switches $M_{1 n}$ and $M_{2 n}$

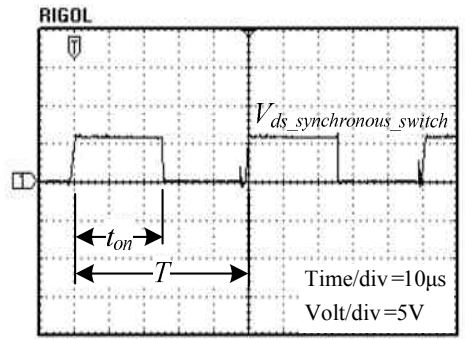

(g) The voltage stress drop across the synchronous active switch

Fig. 9. Oscillograms of the proposed converter.

B3, is shown in Fig. 9(b). The currents of the batteries with a moderate voltage, $B_{2}$ and $B_{1}$, are shown in Fig. 9(c) and Fig. 9(d), respectively. The AC-linked voltage at the interconnection bus is shown in Fig. 9(e).

The voltage stress drop across the main active switches $M_{1 n}$ and $M_{4 n}$ is shown Fig. 9(f). $M_{2 n}$ and $M_{3 n}$ were set to a synchronous switch, and the voltage stress drop across the synchronous active switch is shown Fig. $9(\mathrm{~g})$. At the near end of period (T) in Fig. 9(e) and 9(f), the AC-linked voltage and the voltage drop across the active switch $\left(V_{d s}\right)$ increased slightly because this region had no synchronous signal driving the synchronous switches $\left(M_{2 n}\right.$ and $\left.M_{3 n}\right)$. Both the voltage stress and the voltage spike of the primary switch were low, suggesting that a low-voltage component for this circuit could be used. In commercial terms, the use of a

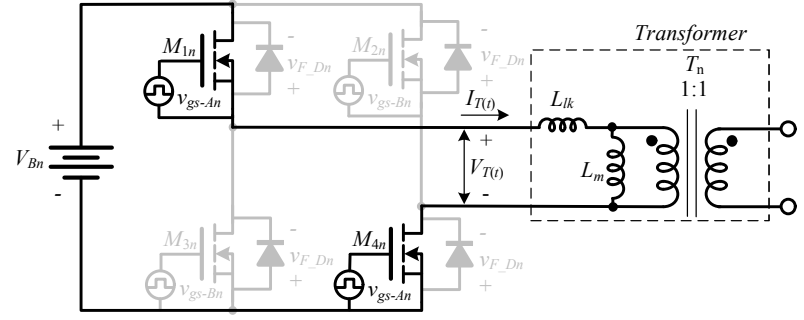

(a) For the operation at first stage

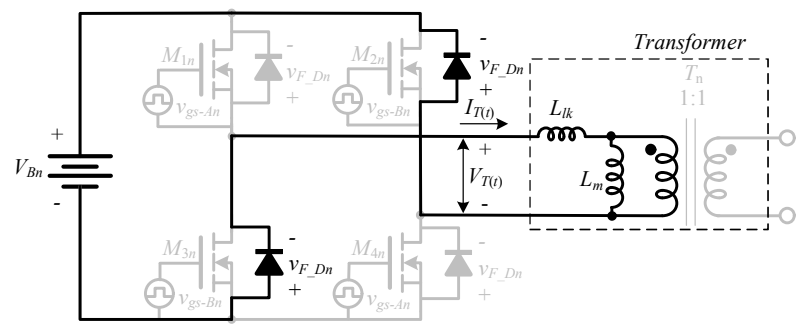

(b) The operation during a dead time period

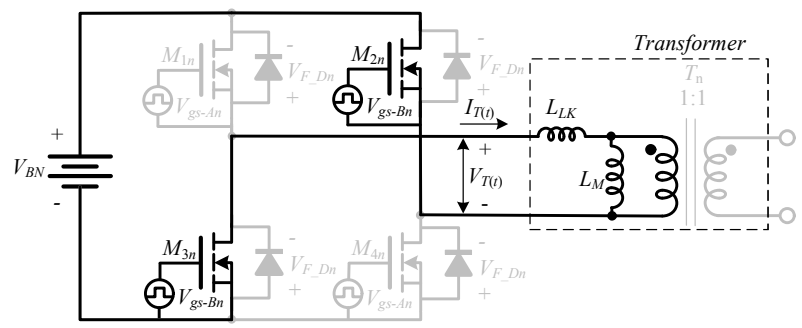

(c) The operation at the second stage

Fig. 10. The operation of the proposed circuit that cause switching current and voltage spikes.

low-voltage component is more economical, and the reliability of a low-stress system is higher.

Considering the current and voltage oscillograms of the proposed converter, it was found that the switching devices are facing off with a high current and AC-linked voltage high voltage spikes. These spikes occurred because of the dead time interval between the first operating $\left(t_{0}-t_{1}\right)$ and second operating stages $\left(t_{1}-t_{2}\right)$. The dead time interval was necessary to prevent a short circuit condition between the high-side and low-side switches of the converter and this could not be neglected. Upon first operating, stages $M_{1 n}$ and $M_{2 n}$ were synchronously turned on, and the magnetizing flux increased. The primary transformer voltage $V_{T(t)}$ was equal to $V_{B n}$, as shown in Fig. 10(a).

After finishing the first operating stage, $M_{1 n}$ and $M_{2 n}$ were synchronously turned off. Then the dead time interval started, as shown in Fig. 10(b). In this interval, the magnetizing flux decreased, the primary transformer voltage had inverse polarity and was equal to:

$$
-V_{T(t)}=\left(\left(2 \mathrm{x} V_{F_{-} D n}\right)+V_{B n}\right)
$$

After the dead time period ended, the second stage started 
$T_{\mathrm{n}}$

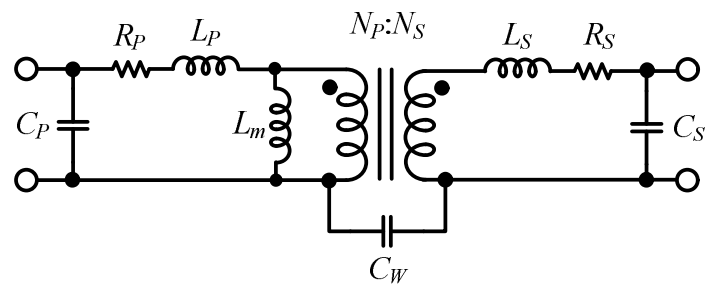

Fig. 11. The parasitic elements of the transformer model in the proposed CES.

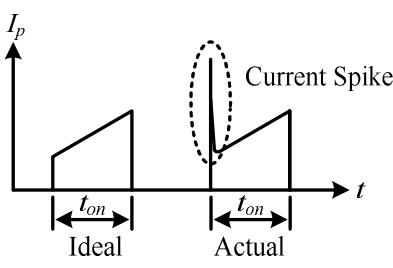

(a) Current spike

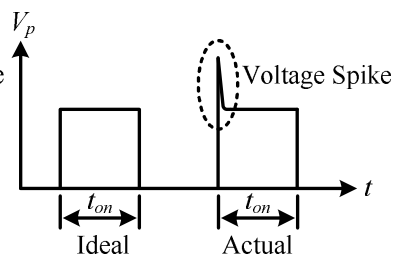

(b) Voltage spike
Fig. 12. The spikes at the switching device.

while the primary transformer voltage was equal to (11), and switches $M_{2 n}$ and $M_{3 n}$ were synchronously turned on. At the beginning of this second stage the current spike occurred for a short period of time because the primary transformer voltage had a higher potential than the normal stage. Then the primary transformer voltage $-V_{T(t)}$ decreased (from (11)) to equal $V_{B n}$. The current of the inductor slowly decreased to a normal operating stage, as shown in Fig. 10(c).

In the proposed circuit, the switching transformer with a ratio of $N_{P}: N_{S}$, equaled $1: 1$ and consisted of many parasitic elements. These elements of are shown in Fig. 11. This was measured using a LCR meter (Model LCR817, GWINSTEK).

The parasitic measurement results were $R_{P}=0.103 \mathrm{~m} \Omega, R_{S}$ $=0.103 \mathrm{~m} \Omega, L_{P}=1.13 \mu \mathrm{H}, L_{S}=1.21 \mu \mathrm{H}, L_{m}=165 \mu \mathrm{H}$, and $C_{W}$ $=0.212 \mathrm{nF} . C_{P}$ and $C_{S}$ are very small and they can be neglected.

One cause of the current spike was the winding capacitance $C_{W}$ coupling between primary winding and the secondary winding [37]. It caused a small current spike at the main active switching device, as shown in Fig 12(a). On the other hand, the leakage inductance of the transformer caused a voltage spike across the main active switching device, as shown in Fig 12(b).

These voltage and current spikes in general were generated by the behavior of the proposed circuit. To reduce the current spike, a Schottky diode was installed parallel to the internal diode of the active switching device in order to reduce $V_{F_{-} D n}$. It could also be reduced by using a resonant-mode converter, which eliminates the voltage and current spike problems of the charge equalized module. However, this is a topic of future research.

The efficiency of the average energy transferred by the proposed technique is shown in Fig. 13.

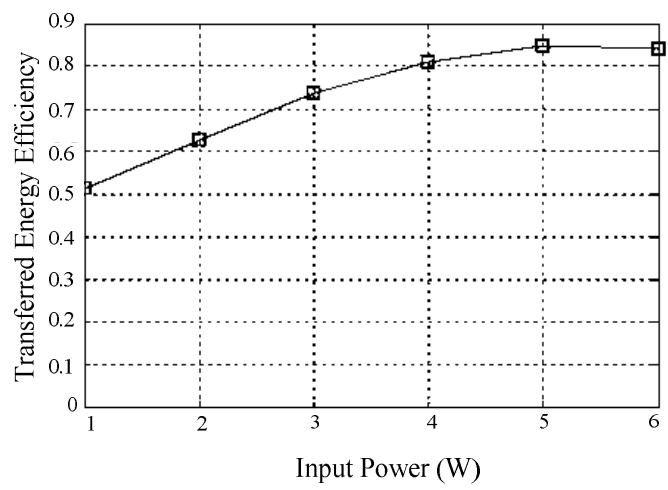

Fig. 13. The efficiency of the average energy transferred by the proposed technique.

The procedure used to measure the converter-transferred energy efficiencies, shown in Fig. 14(a), involved the following steps:

- Supply a constant voltage $(7 \mathrm{~V})$ to the first converter module.

- Connect a load resistance of $6.2 \Omega$ at the output terminal of the fourth converter module.

- Open the terminal circuit of the second and third converter modules.

- Vary the duty cycle command for the active switch of the first converter.

- The synchronous signal generator generates a synchronous command to the synchronous switches $D_{1 n}$ and $D_{2 n}$.

As a result, the maximum efficiency of the proposed technique was $85 \%$ at a power output of $5 \mathrm{~W}$ (calculated from $P_{o} / P_{\text {in }}$ of the converter $P_{\text {in }}=V_{S} I_{\text {in }}$ and $P_{\text {out }}=V_{\text {out }} I_{\text {out }}$ ). If the power input came from battery $B_{1}$ and the output went to battery $B_{4}$, the efficiency was then equal to $V_{B 4} I_{B 4} / V_{B 1} I_{B 1}$

Fig. 14(b) shows a comparison of different methods for the transfer of excess energy from battery $B_{4}$ to battery $B_{1}$. Using the DC-linked charging technique [33] to transfer excess energy from battery $B_{4}$ to battery $B_{1}$, made it necessary to operate the converters of at least two modules at the same time. The first module converted excess energy from the battery to the DC-linked voltage bus, and the second module converted the DC-linked voltage and transferred it to the target battery. Conversely, the AC-linked technique had the ability to transfer energy from a source battery to a target battery in a single shot. This can increase the total transferred energy efficiency.

The work of Ref. [33] used a converter with an efficiency of $80 \%$, which means that the total efficiency of the energy transferred was $(0.8 \times 0.8) \times 100 \%=64 \%$. When compared with the proposed topology the efficiency is increased by more than $21 \%$ over the DC-linked bus topology. 


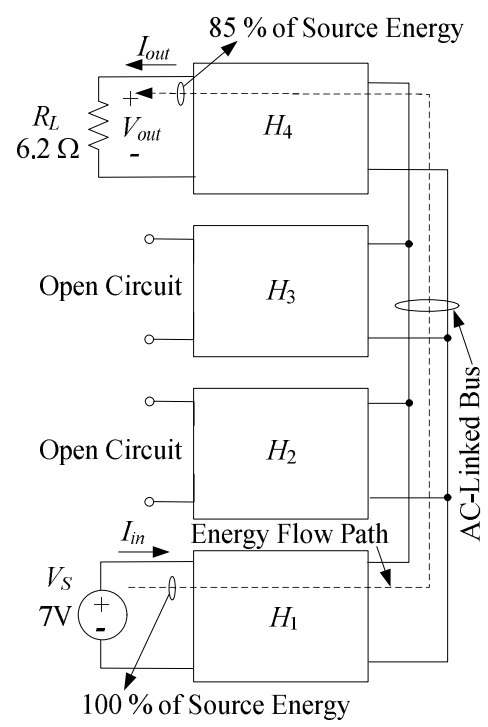

(a) AC-linked technique

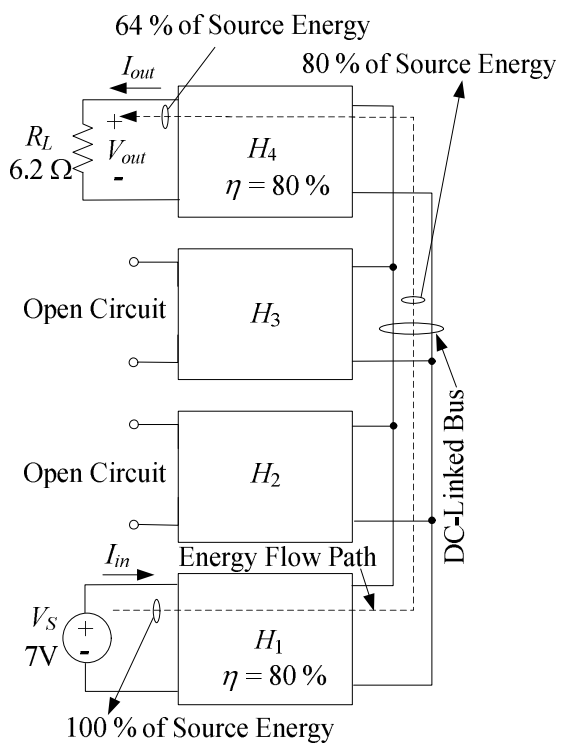

(b) DC-linked technique

Fig. 14. Diagram used to measure the converter-transferred energy efficiency.

\section{CONCLUSION}

The proposed low-voltage-stress AC-linked charge equalizing system satisfactorily improved the overall energy transfer efficiency. It was practical to implement a high-efficiency battery management system by incorporating a charge equalization circuit (CEC) and a microcontroller into the battery equalization process. This converter-controller combination eliminated the problem of unbalanced battery voltages. With a modularized design, embedded with a microcontroller, the proposed system was compatible with any number of batteries in the SCBS. However, to take full advantage of the proposed topology, the number of batteries in the SCBS should be larger than 3. The experimental results showed that the efficiency when using the CEC was $85 \%$ at an average power output of $5 \mathrm{~W}$. The proposed technique had a modularized design that was simple to construct, install and maintain. The main disadvantage of the proposed topology was a complicated switch driving circuit. This circuit also faced a high current stress when the number of CESs was expanded to a larger scale SCBS. In the future, a single-stage converter with a high power output capability, a one-cycle control and the ability to operate in resonant mode will be studied. The advantages of such a system could be fast control and applicability to linear and nonlinear situations.

\section{ACKNOWLEDGEMENTS}

We would like to thank the Department of Electronic and Telecommunication Engineering and the Department of Computer Engineering, Faculty of Engineering, King Mongkut's University of Technology Thonburi, Bangkok, Thailand, for providing funds to partially support this study. We would also like to thank Dr. Rainer Zawadzki (RMUTL) for advice on the manuscript.

\section{REFERENCES}

[1] Panasonic, Valve regulated lead acid VRLA OEM batteries (2010), http://www.panasonic.com/industrial/ batteries-oem/ oem/lead-acid-vrla.aspx.

[2] B. B. battery technical manual (2010), http://www.bbbattery.com/techmanual.asp.

[3] B. Lindemark: "Individual cell voltage equalizers (ICE) for reliable battery performance," INTELEC ' $91,13^{\text {th }}$ International Conference, pp. 196-201, 1991.

[4] M.-W. Cheng, S.-M. Wang, Y.-S. Lee, and S.-H. Hsiao, "Fuzzy controlled fast charging system for lithium-ion batteries," PEDS, pp. 1498-1503, 2009.

[5] S. Bergvik and L. Bjorkstrom, "Prolonged useful life and reduced maintenance of lead-acid batteries, by means of individual cell voltage regulation," INTELEC, pp. 63-66, 1984.

[6] D. Bjork, Maintenance of batteries: "New trends in batteries and automatic battery charging," INTELEC, pp. 355-360, 1986.

[7] N. H. Kutkut, H. L. N. Wiegman, D. M. Divan, and D. W. Novotny: "Design considerations for charge equalization of an electric vehicle battery system," APEC, Vol. 1, pp. 96-103, 1995.

[8] N. H. Kutkut, H. L. N. Wiegman, D. M. Divan, and D. W. Novotny: "Design considerations for charge equalization of an electric vehicle battery system," IEEE Trans. Ind. Appl., Vol. 35, No. 1, pp. 28-35, Jan./Feb. 1999.

[9] T.-Z. Wu, Y. Sun, H.-J. Zhou, and F.-Z. Xia: "Study on the equalizing charge of serial Ni-MH traction batteries," ICECE, pp. 4325-4328, 2010.

[10]H. Sakamoto, K. Murata, K. Nishijima, K. Harada, S. Taniguchi, K. Yamasaki, and G. Ariyoshi: "Balanced charging of series connected battery cells," INTELEC, pp. 311-315, 1998.

[11]Y. C. Hsieh, C. S. Moo, and W. Y. Ou-Yang, "A bi-directional charge equalization circuit for series-connected batteries," PEDS, pp. 1578-1583, 2005.

[12]C. Pascual and P. T. Krein, "Switched capacitor system for automatic series battery equalization," $A P E C$, Vol. 2, pp. 
848-854, 1997.

[13] S. West and P. T. Krein, "Equalization of valve-regulated lead-acid batteries: issues and life test results," INTELEC, pp. 439-446, 2000.

[14]A. Baughman and M. Ferdowsi, "Analysis of the double-tiered three-battery switched capacitor battery balancing system," $V P P C$, pp. 1-6, 2006.

[15]A. C. Baughman and M. Ferdowsi, "Double-tiered switched-capacitor battery charge equalization technique," IEEE Trans. Ind. Electron., Vol. 55, No. 6, pp. 2277-2285, Jun. 2008 .

[16]H.-S. Park, C.-H. Kim, K.-B. Park, G.-W. Moon, and J.-H. Lee, "Design of a charge equalizer based on battery modularization," IEEE Trans. Veh. Technol., Vol. 58, No. 7, pp. 3216-3223,Sep. 2009.

[17] Y. S. Lee, S. H. Chen, and Y. P. Ko, "Micro-Controller Unit Application in fuzzy battery equalization control for battery string," SMC, Vol. 3, pp. 2110-2115, 2006.

[18] Y. C. Hsieh, C. S. Moo, and I. S. Tsai, "Balance charging circuit for charge equalization," Power Conversion Conference, Vol. 3, pp. 1138-1143, 2002.

[19]C. Karnjanapiboon, Y. Rungruengphalanggul, and I. Boonyaroonate: "The low stress voltage balance charging circuit for series connected batteries based on buck-boost topology," ISCAS, Vol. 3, pp. III-284-III-287, 2003.

[20]Y.-S. Lee and C.-W. Jao, "Fuzzy controlled lithium-ion battery equalization with stage-of-charge estimator," SMC International Conference, Vol. 5, pp. 4431-4438, 2003.

[21] Y.-S. Lee and M.-W. Cheng, "Intelligent control battery equalization for series connected lithium-ion battery strings," IEEE Trans. Ind. Electron., Vol. 52, No. 5, pp. 1297-1307, Oct. 2005.

[22] C.-H. Kim, H.-S. Park, C.-E. Kim, G.-W. Moon, J.-H. Lee, and J. K. Oh, "Charge equalization converter with parallel primary winding for series connected Lithium-Ion battery strings in HEV," ICPE International Conference, pp. 795-800, 2007.

[23]H.-S. Park, C.-E. Kim, C.-H. Kim, B.-C. Kim, G.-W. Moon, and J.-H. Lee, "Modularized charge equalization converter with high power density and low voltage stress for HEV lithium-ion battery string," ICPE International Conference, pp. 784-789, 2007.

[24]C.-H. Kim, H.-S. Park, and G.-W. Moon, "A modularized two-stage charge equalization converter for series connected lithium-ion battery strings in an HEV," PESC, pp. 992-997, 2008.

[25]H.-S. Park, C.-H. Kim, and G.-W. Moon, "Charge equalizer design method based on battery modularization," ICSET International Conference, pp. 558-563, 2008.

[26]C.-H. Kim, M.-Y. Kim, J.-H. Kim, and G..-W. Moon, "Modularized charge equalizer with intelligent switch block for lithium-ion batteries in an HEV," INTELEC, pp. 1-6, 2009.

[27]C.-H. Kim, Y.-D. Kim, G..-W. Moon, and H.-S. Park, "Individual cell voltage equalizer using selective two current paths for series connected li-ion battery strings," ECCE, pp. 1812-1817, 2009.

[28]H.-S. Park, C.-E. Kim, C.-H. Kim, G.-W. Moon, and J.-H. Lee, "A modularized charge equalizer for an HEV Lithium-Ion battery string," IEEE Trans. Ind. Electron., Vol. 56, No. 5, pp. 1464-1476, May 2009.

[29] S.-M. Wang, M.-W. Cheng, Y.-S. Lee, R.-H. Chen, and W.-T. Sie, "Intelligent charged system for Lithium-ion battery strings," INTELEC, pp. 1-6, 2009.

[30] J. Yan, Z. Cheng, G. Xu, H. Qian, and Y. Xu, "Fuzzy control for battery equalization based on stage of charge," VTC Conference, pp. 1-7, 2010.
[31]C. Karnjanapiboon, K. Jirasereeamornkul, and V. Monyakul: "High efficiency battery management system for serially connected battery string," ISIE, pp. 1504-1509, 2009.

[32] C. Karnjanapiboon, K. Jirasereeamornkul, and V. Monyakul: "Charge equalized module for serially connected VRLA battery string using quasi-resonant flyback converter," ICIEA, pp. 2148-2153, 2010.

[33] C. Karnjanapiboon, K. Jirasereeamornkul, and V. Monyakul: "The high efficiency charge equalized system for serially connected VRLA battery string using synchronous flyback converter," IPEC, pp. 1185-1188, 2010.

[34]M. Einhorn, W. Roessler, and J. Fleig: "Improved performance of serially connected Li-Ion batteries with active cell balancing in electric vehicles," IEEE Trans. Veh. Technol., Vol. 60, No. 6, pp. 2448-2457, Jul. 2011.

[35]H. S. Park, C. E. Kim, C. H. Kim, G. W. Moon, and J. H. Lee, "A modularized charge equalization converter for a hybrid electric vehicle lithium-ion battery stack," Journal of Power Electronics, Vol. 7, No.4, pp. 343-352, Oct. 2007.

[36]H. S. Park, C. E. Kim, C. H. Kim, G.. W. Moon, and J. H. Lee, "Individual charge equalization converter with parallel primary winding of transformer for series connected Lithium-Ion battery strings in an HEVM," Journal of Power Electronics, Vol. 9, No.3, pp. 472-480, May 2009.

[37] W. M. T. Mclyman, Transformer and Inductor Design Handbook, $3^{\text {rd }}$, Marcel Dekker, pp. 447-449, 2004.

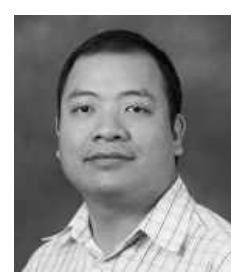

Charnyut Karnjanapiboon was born in Nan, Thailand, in 1980. He received his B.S. in Power Electronic Technology from Mongkut's Institute of Technology North Bangkok (KMITNB), Thailand, in 2002, and his M.S. in Electrical Engineering from Mongkut's University of Technology Thonburi (KMUTT), Bangkok, Thailand, in 2004. His current research interests include power electronics, photo voltaic systems and energy storage systems.

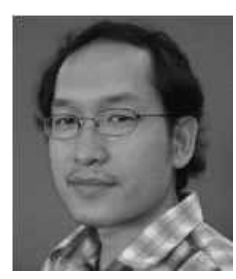

Kamon Jirasereeamornkul was born in Phuket, Thailand, in 1975. He received his B.S. and M.S. in Electrical Engineering and his Ph.D. in Electrical and Computer Engineering from King Mongkut's University of Technology Thonburi (KMUTT), Bangkok, Thailand, in 1997, 2001 and 2006, respectively, where he is currently working as Lecturer in the Department of Electronic and Telecommunication Engineering, Faculty of Engineering, KMUTT. His current research interests include high-frequency power converters, high-frequency inverters and electronic ballasts.

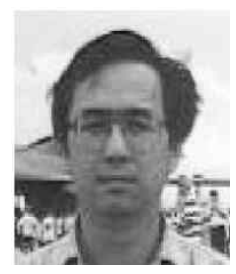

Veerapol Monyakul was born in Thailand. $\mathrm{He}$ received his Ph.D. in Electronic Engineering from Oklahoma State University, USA, in 1993. He is presently is a Researcher in the Biochemical Engineering and Pilot Plant Research and Development Unit (BEC), National Science and Technology Development Agency (NSTDA), Thailand. His current research interests include energy technology and driving systems. 